\title{
Surface Relaxation Below the Roughening Temperature: Some Recent Progress and Open Questions
}

\author{
Robert V. Kohn* \\ Courant Institute of Mathematical Sciences \\ New York University \\ kohn@cims.nyu.edu
}

December 2010

To appear in the Proceedings of the 2010 Abel Symposium on Nonlinear Partial Differential Equations (Oslo, Sept 28-Oct 1, 2010)

\begin{abstract}
We discuss two recent projects concerning the evolution of a crystal surface below the roughening temperature. One addresses the evolution of a monotone one-dimensional step train (joint work with Hala Al Hajj Shehadeh and Jonathan Weare). The other addresses the finite-time flattening predicted by a fourth-order PDE model (joint work with Yoshikazu Giga). For each project we begin with a discussion of the mathematical model; then we summarize the recent results, the main ideas behind them, and some related open problems.
\end{abstract}

\section{Introduction}

The surface of a crystal below its roughening temperature consists of steps and terraces, with facets at the peaks and valleys. The steps interact and collide, and if the average slope is zero then surface relaxes to a perfectly flat state (a single facet). This process can be modeled at the atomic scale using a kinetic Monte Carlo scheme. But Monte Carlo is limited to relatively small length and time scales. To understand and simulate the macroscopic consequences of relaxation, it is therefore attractive to use either

- step evolution laws, which ignore atomic-scale fluctuations and track instead the positions of steps; or

- partial differential equations, which model the evolution on a coarser, continuum scale.

${ }^{*}$ This research was supported by NSF through grant DMS-0807347. 
For a given physical system there is often considerable uncertainty which approach is best. Therefore it is important to understand the models' qualitative predictions. Exploration of this issue leads to a wide array of mathematical questions, many of which remain open. The present article summarizes recent progress on two problems of this type:

(a) work with H. Al Hajj Shehadeh and J. Weare on the asymptotic self-similarity of a monotone one-dimensional step train in the attachment-detachment-limited (ADL) regime [1], and

(b) work with Y. Giga on the finite-time flattening predicted by a fourth-order PDE describing relaxation by surface diffusion in the diffusion-limited (DL) regime [8].

We also highlight a number of open questions of an essentially mathematical character, which we think may be ripe for progress. For broader surveys of the physics of crystal growth and relaxation we refer to the recent monographs $[18,23]$ and the review article [12].

\section{A monotone one-dimensional step train in the ADL regime}

For a two dimensional surface with peaks and valleys, the analysis of step motion is complicated because (i) each step is a curve with a different shape, and (ii) the steps collide at peaks and valleys. Therefore it is natural to start with a simpler problem: a one-dimensional step train connecting two semi-infinite terraces as in Figure 1. We take the convention that there are $N$ steps, each of height $1 / N$. The semi-infinite terrace to the left of $x_{1}$ has height 0 , and the one to the right of $x_{N}$ has height 1 .

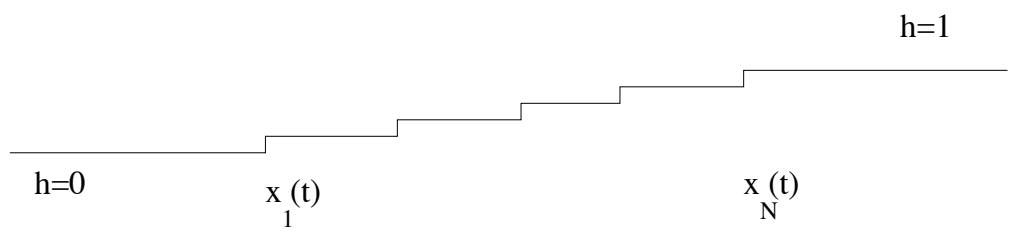

Figure 1: A monotone one-dimensional step train.

After discussing the steps' motion law, we shall explain why the evolution is asymptotically self-similar as $t \rightarrow \infty$, and we'll discuss the anticipated behavior in the continuum limit $N \rightarrow \infty$.

\subsection{The step equations}

Ozdemir and Zangwill [22] were, it seems, the first to identify the step velocities in this setting by specializing the framework of Burton, Cabrera, and Frank [3] (a special case was considered a few years earlier by Rettori and Villain [24]). Their calculation is reviewed e.g. in [19] and in Appendix A of [1]. Away from the extremes, the velocity of the $i$ th step is

$$
\dot{x}_{i}=c\left(\frac{\mu_{i+1}-\mu_{i}}{\left(x_{i+1}-x_{i}\right)+2 D / k}-\frac{\mu_{i}-\mu_{i-1}}{\left(x_{i}-x_{i-1}\right)+2 D / k}\right)
$$


where $c$ is a (dimensional) constant,

$$
\mu_{i}=\left(x_{i+1}-x_{i}\right)^{-3}-\left(x_{i}-x_{i-1}\right)^{-3},
$$

and $D / k$ is the ratio of the terrace diffusion constant $D$ to a kinetic coefficient $k$ with the dimensions of length/time. For the second step $(i=2)$ and the second-last step $(i=N-1)$ the velocity is still given by (1), but the definitions of $\mu_{1}$ and $\mu_{N-1}$ are

$$
\mu_{1}=\left(x_{2}-x_{1}\right)^{-3}, \quad \mu_{N-1}=-\left(x_{N}-x_{N-1}\right)^{-3} .
$$

For the first and last step, the velocities are

$$
\dot{x}_{1}=c \frac{\mu_{2}-\mu_{1}}{\left(x_{2}-x_{1}\right)+2 D / k}, \quad \dot{x}_{N}=-c \frac{\mu_{N}-\mu_{N-1}}{\left(x_{N}-x_{N-1}\right)+2 D / k} .
$$

Note that (3) is consistent with (2) if we take $x_{0}=-\infty$ and $x_{N+1}=\infty$. The formulas for $\dot{x}_{1}$ and $\dot{x}_{N}$ have just one term rather than two, because the extreme steps receive diffusing atoms only from one side.

\subsection{A convenient reorganization}

As Ozdemir and Zangwill pointed out, it is convenient to consider the discrete slopes

$$
u_{i}=\frac{1 / N}{x_{i+1}-x_{i}}, \quad 1 \leq i \leq N-1
$$

and to view the second order difference operator

$$
\Delta_{i} \xi=N^{2}\left(\xi_{i+1}-2 \xi_{i}+\xi_{i-1}\right)
$$

as the discrete Laplacian with respect to height. Then the step equations (1)-(4) reduce by algebraic manipulation to

$$
\dot{u}_{i}=-c N u_{i}^{2} \Delta_{i}\left[\frac{u}{1+\frac{2 D N}{k} u} \Delta u^{3}\right], \quad 1 \leq i \leq N-1,
$$

with the conventions

$$
u_{0}=u_{N}=0 \quad \text { and } \quad \Delta_{0} u^{3}=\Delta_{N} u^{3}=0 .
$$

\subsection{The DL and ADL regimes}

The ratio $D / k$ has the dimensions of length. So does $1 / N$, since it is the height of a single step. Therefore $D N / k$ is a dimensionless ratio. When it is large, diffusion across terraces is rapid, and the attachment or detachment of atoms at steps sets the timescale of step motion; this is called the attachment-detachment-limited (ADL) regime. Conversely, when $D N / k$ is small, attachment or detachment at steps is rapid, and it is the diffusion of atoms across terraces that sets the timescale of step motion; this is diffusion-limited (DL) regime. Evidently, the step motion laws reduce in these two limits (after an appropriate rescaling of time) to

$$
\begin{aligned}
& \dot{u}_{i}=-u_{i}^{2} \Delta_{i}\left[\Delta\left(u^{3}\right)\right] \quad \text { in the ADL regime, and } \\
& \dot{u}_{i}=-u_{i}^{2} \Delta_{i}\left[u \Delta\left(u^{3}\right)\right] \quad \text { in the DL regime. }
\end{aligned}
$$




\subsection{Self-similar asymptotics in the ADL regime}

Ozdemir and Zangwill observed numerically that as $t \rightarrow \infty$, solutions of the ADL evolution law (9) are asymptotically self-similar. Our recent paper [1] provides the first rigorous analysis, showing that:

- There is a unique positive self-similar solution of (9), which by definition has the form

$$
u_{i}(t)=t^{-1 / 4} \phi_{i}
$$

with $\left(\phi_{1}, \ldots, \phi_{N-1}\right)$ independent of $t$.

- A solution of (9) with positive initial data remains positive for all $t$, and is asymptotically self-similar in the sense that

$$
t^{1 / 4} u_{i}(t) \rightarrow \phi_{i} \quad \text { as } t \rightarrow \infty
$$

- The self-similar profile $\left(\phi_{1}, \ldots \phi_{N-1}\right)$ is the unique positive minimizer of

$$
S E_{N}[w]=\sum_{i=1}^{N-1}-\frac{1}{8} w_{i}^{2}+\frac{1}{6}\left[\Delta_{i}\left(w^{3}\right)\right]^{2}
$$

subject to $w_{0}=w_{N}=0$. Moreover, the graph of $\phi_{i}^{3}$ is concave in the sense that $\Delta_{i}\left(\phi^{3}\right)<0$ for $1 \leq i \leq N-1$.

The key to these results is the observation that the ADL evolution law (9) is $L^{2}$ steepest descent for

$$
E_{N}=\sum_{i=1}^{N-1} \frac{1}{6}\left(\Delta_{i} u^{3}\right)^{2}
$$

subject to $u_{0}=u_{N}=0$. Changing to "similarity variables," i.e. writing

$$
u_{i}(t)=t^{-1 / 4} w_{i}(s) \quad \text { with } s=\log t,
$$

one finds that the slope evolution (9) is equivalent to

$$
\frac{d w_{i}}{d s}=\frac{1}{4} w_{i}-w_{i}^{2} \Delta_{i} \Delta\left(w^{3}\right),
$$

which amounts to $L^{2}$ steepest descent for the functional $S E_{N}$ defined by (13). Now, $S E_{N}$ has a unique positive critical point, because if we write $v_{i}=w_{i}^{3}$ then each of its two terms is a convex function of $v$. In addition, one can show (using energy-type inequalities) that the solution "in similarity variables" $w_{i}(s)$ remains strictly positive and bounded for all time. Asymptotic self-similarity follows, since the $\omega$-limit set of a steepest descent consists of critical points of its Lyapunov functional. 


\subsection{The continuum limit of the ADL law}

Recall from (6) that $\Delta_{i}$ is the centered finite-difference Laplacian with respect to height. Therefore the continuum analogue of the ADL evolution law (9) is (at least formally)

$$
u_{t}=-u^{2}\left(u^{3}\right)_{h h h h} \quad \text { for } 0<h<1,
$$

with boundary conditions $u(t, 0)=u(t, 1)=0$ and $\left(u^{3}\right)_{h h}(t, 0)=\left(u^{3}\right)_{h h}(t, 1)=0$. This PDE is degenerate-parabolic wherever $u$ approaches 0 , which must happen at least at the endpoints $h=0,1$. We do not know whether (17) has a well-defined, unique solution (to which our discrete evolution should presumably converge as $N \rightarrow \infty$ ).

The continuum self-similar solution is, however, more accessible: since the discrete self-similar solution minimizes the discrete "energy" defined by (13), the continuum self-similar solution should minimize its continuous analogue

$$
S E[w]=\int_{0}^{1}-\frac{1}{8} w^{2}+\frac{1}{6}\left[\left(w^{3}\right)_{h h}\right]^{2} d s
$$

subject to $w(0)=w(1)=0$. We studied this variational problem in [1], showing that

(i) The functional $S E$ has a unique positive minimizer $\phi$. Moreover, $\phi^{3}$ is $C^{3, \alpha}$ on the closed interval $[0,1]$, and it vanishes linearly at each endpoint in the sense that $\left(\phi^{3}\right)_{h}(0)=-\left(\phi^{3}\right)_{h}(1)>0$. In addition, $\phi^{3}$ is a concave function of $h$.

(ii) The discrete self-similar solution $\phi_{N}$ converges to the continuous one $\phi$ as $N \rightarrow \infty$, with (roughly speaking) $\left\|\left(\phi_{N}^{3}\right)_{h h}-\left(\phi^{3}\right)_{h h}\right\|_{L^{2}} \leq C N^{-5 / 6}$.

The arguments for (i) are parallel to those used in the discrete setting. The $C^{3, \alpha}$ regularity of $\phi^{3}$ comes from the Euler-Lagrange equation (the continuum analogue of (16) specialized to a steady state)

$$
\left(\phi^{3}\right)_{h h h h}=\frac{1}{4} \phi^{-1}
$$

combined with the concavity of $\phi^{3}$ and its linear behavior near $h=0,1$. The arguments for (ii) use the convexity of $S E$ when viewed as a function of $v=w^{3}$.

We suppose the continuum limit of the time-dependent evolution should resemble that of the similarity solution; in particular, we suppose the solution of (17) should have the property that $u^{3}$ is $C^{3, \alpha}$ up to the boundary, vanishing linearly near $h=0,1$. Since $u$ gives the slope as a function of height and time, one must integrate to get the height as a function of space and time. The length of the region occupied by the steps is

$$
\left.x\right|_{h=1}-\left.x\right|_{h=0}=\int_{0}^{1} x_{h} d h=\int_{0}^{1} \frac{1}{u} d h,
$$

which is finite provided (as proved for the similarity solution) $1 / u$ is integrable on $[0,1]$. Thus, the continuum limit should consist of a monotone, asymptotically self-similar profile connecting two semi-infinite facets. 


\subsection{Some open problems}

We have concentrated on the ADL evolution law (9), because the analysis in [1] is restricted to it. But solutions of the DL evolution law (10) also appear numerically to be self-similar [22]. The decay rate is $t^{-1 / 5}$, and the DL evolution "in similarity variables" is obtained by substituting $u_{i}(t)=t^{-1 / 5} v_{i}(s)$ with $s=\log t$ into the diffusion-limited evolution law (10). This leads to the DL analogue of (16):

$$
\frac{d v_{i}}{d s}=\frac{1}{5} v_{i}-v_{i}^{2} \Delta_{i}\left[v \Delta\left(v^{3}\right)\right]
$$

Unfortunately, we do not know a steepest-descent interpretation of the DL evolution. Therefore the methods we used for the ADL case seem not to be applicable in the DL setting. (The recent paper by Nakamura and Margetis [17] examines the DL self-similar solution using an entirely different technique, but does not address its stability.)

Our paper [1] considers only a monotone step train connecting two semi-infinite terraces as in Figure 1. But there are also other examples involving monotone step trains where the step dynamics appears to be asymptotically self-similar. This was pointed out by Israeli, Jeong, Kandel, and Weeks [9], who considered several cases including

(a) relaxation of an infinite bunch - directly analogous to the example discussed above, but with a terrace only on one side (Figure 2, right); and

(b) reconstruction-driven faceting - in which two infinite step trains of the same sign are repelled by an energetically-preferred terrace (Figure 2, left).

The simulations and analysis in [9] (which considers the ADL regime and evaporation-condensation dynamics) shows convincingly that the solutions are asymptotically self-similar. We wonder whether the methods of [1] might suffice to give a proof, and perhaps to give a variational characterization of the similarity solution.
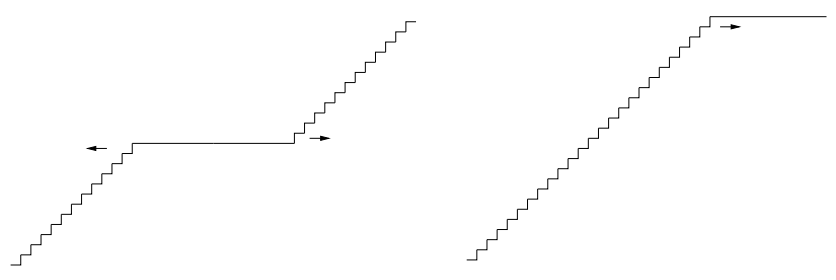

Figure 2: Initial conditions for two examples considered in [9]. Left: reconstruction-driven faceting. Right: relaxation of an infinite bunch.

A monotone step train has the advantage that its steps don't collide, so the maximum and minimum heights don't change with time. The relaxation of a rough profile - for example a periodic one, like Figure 3 - is fundamentally different, because collision of steps is the crucial mechanism by which the maximum height decreases and the minimum increases, leading eventually to a single flat facet. But perhaps the self-similarity of monotone profiles can be used to understand this process. Indeed, Ozdemir and Zangwill argue in [22] that each monotone region will be close to a self-similar 
solution, and they use this idea to predict the times when steps collide and the overall flattening rate. Such an argument was explored further by Israeli and Kandel [11], who permitted steps of opposite sign to interact. We wonder whether these arguments can be made rigorous. A natural first step might be to consider the flattening of an infinite groove - another example from [9]. Similar issues arise in the radial setting $[5,10]$. The paper [11] also raises an interesting modeling question: can one determine, by an atomic-scale or Burton-Cabrera-Frank-type argument, how steps of opposite sign should interact?

This section has focused on one-dimensional step models. Some authors have questioned the physical appropriateness of such models, arguing that two-dimensional fluctuations near a peak or valley cannot be ignored. A good discussion of this topic can be found in [28].

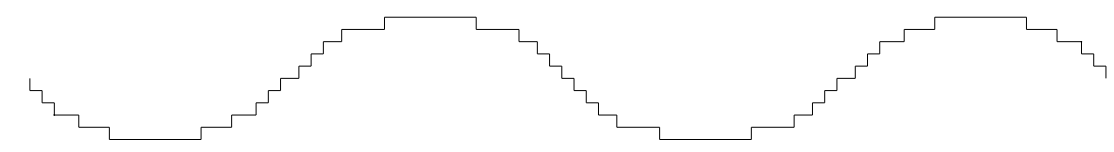

Figure 3: In a periodic step-train, steps collide at peaks and valleys.

\section{A thermodynamic PDE model}

The approach discussed in Section 2 starts with a step motion law, and considers its continuum limit by taking $N \rightarrow \infty$. There is also another, more phenomenological viewpoint, in which the continuum surface height solves a fourth-order PDE of the form

$$
h_{t}=-\operatorname{div}\left[M(\nabla h) \nabla \operatorname{div}\left(\beta_{1} \frac{\nabla h}{|\nabla h|}+\beta_{3}|\nabla h| \nabla h\right)\right]
$$

with $\beta_{1}, \beta_{3}>0$. For spatially periodic structures the boundary condition should of course be periodic. For spatially complex but statistically homogeneous structures it is again natural to use periodic boundary conditions, with a large enough period cell. Therefore we shall focus in the following discussion on the periodic setting (though the paper [8] also considers some other cases). On the facets, where $\nabla h=0$, the PDE (20) requires interpretation; we shall comment on this below. We always assume that the initial data has mean value 0 ; since $h_{t}$ is a divergence, the mean remains 0 for all $t$.

As time increases, the surface should relax to a perfectly flat state (a single facet). The rate at which it flattens can often be observed experimentally. Therefore it is natural to ask what the models predict in this regard. One-dimensional simulations with sinusoidal initial data and $M(\nabla h)=1$ (the DL regime, see below) indicate $[7,26]$ that when $\beta_{1}>0$, the solution becomes identically zero at a finite time $T^{*}$, and $h_{\max } \sim\left(T^{*}-t\right) \lambda^{-3}$ where $\lambda$ is the period size. Some analytical results supporting this conclusion can be found in [21, 25].

After briefly summarizing the logic behind (20) we shall discuss our recent work [8], which addresses flattening behavior of the PDE when $M=1$ and $\beta_{1}=1$. Our main result is an estimate for the flattening time, which shows (at the level of an inequality) that the general case (for any initial data, in any space dimension) is similar to the $1 \mathrm{D}$, sinusoidal setting. 


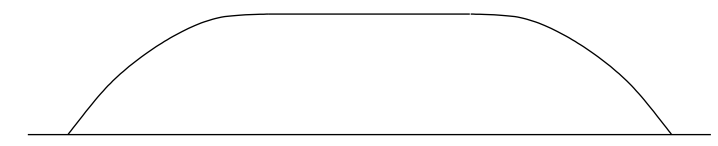

Figure 4: Solution of the isoperimetric problem (22).

Numerical results are also available in the ADL setting, and when $\beta_{1}=0$. We shall discuss them briefly in Section 3.4.

\subsection{Background}

The PDE (20) is, roughly speaking, obtained by applying a "thermodynamic" viewpoint to the singular surface energy

$$
E[h]=\int \beta_{1}|\nabla h|+\frac{1}{3} \beta_{3}|\nabla h|^{3} .
$$

The thermodynamic viewpoint, which dates back at least to Mullins' work on grain boundary grooving [20], has long been accepted in connection with motion by surface diffusion above the roughening temperature.

When $\beta_{1}>0$ the surface energy density has a conical singularity at $\nabla h=0$. This reflects the hypothesis that the horizontal orientation is a facet. The second term in the energy density is cubic rather than quadratic, to achieve consistency with the 1D step motion law discussed in Section 2. (We explain this consistency below.)

It is instructive to consider the one-dimensional, small-slope version of the isoperimetric problem:

$$
\min \int_{0}^{1} \beta_{1}\left|h_{x}\right|+\frac{1}{3} \beta_{3}\left|h_{x}\right|^{3} d x, \quad \text { subject to } h(0)=h(1)=0 \text { and } \int_{0}^{1} h(x) d x=\text { constant. }
$$

The solution has curved sides and a flat facet at the top, as shown in Figure 4. The edges of the facet are free boundaries, in the sense that their locations are determined by energy minimization.

We are interested in dynamics, not statics. Conservation of mass says

$$
h_{t}+\operatorname{div} j=0
$$

where $j$ is the surface current. The thermodynamic viewpoint says

$$
j=M(\nabla h) \nabla \mu
$$

where $M(\nabla h)$ is a suitable "mobility" and $\mu$ (the "chemical potential") is minus the first variation of $E$ with respect to $h$. When $E$ has the form (21), (23) and (24) combine to give (20).

What is $M(\nabla h)$ ? In one space dimension, the answer can be found by coarse-graining the step velocity law (1) for a monotone step train $[19,22]$. Since $h\left(x_{i}(t), t\right)=$ constant, the step velocity is $\dot{x}_{i}=-h_{t} / h_{x}$. To find the continuum version of the right hand side of (1), we observe that

$$
\frac{\mu_{i+1}-\mu_{i}}{\left(x_{i+1}-x_{i}\right)+2 D / k}=\left(1+\frac{2 D}{k \delta x}\right)^{-1} \frac{\delta \mu}{\delta x}
$$


where $\delta x=x_{i+1}-x_{i}$ and $\delta \mu=\mu_{i+1}-\mu_{i}$. With the approximations

$$
\frac{\delta h}{\delta x} \approx h_{x}, \quad \frac{\delta \mu}{\delta x} \approx \mu_{x}
$$

and remembering that $\delta h=1 / N$, the right hand side of (25) becomes $M\left(h_{x}\right) \mu_{x}$ with

$$
M\left(h_{x}\right)=\left(1+\frac{2 D N}{k} h_{x}\right)^{-1} .
$$

Proceeding in the same spirit, one finds that the continuum version of $\mu$ (defined by (2)) is a constant times $h_{x}^{-1}\left(h_{x}^{3}\right)_{x}=\frac{3}{2}\left(h_{x}^{2}\right)_{x}$, and the continuum limit of the step velocity law becomes (after a suitable change of variables in time)

$$
h_{t}=-\left(M\left(h_{x}\right)\left(h_{x}^{2}\right)_{x x}\right)_{x} .
$$

This is simply (20) with $\beta_{3}=1$. (Notice that in one space dimension, the term in the PDE associated with the singular singular part of the energy $\beta_{1}\left|h_{x}\right|$ vanishes when $h_{x}>0$.)

This calculation is the basis of the widespread view that the mobility in (20) should be

$$
M_{\mathrm{DL}}(\nabla h)=1 \text { and } M_{\mathrm{ADL}}=|\nabla h|^{-1}
$$

in the DL and ADL limits (when $D N / k \rightarrow 0$ or $\infty$ respectively). Actually, the situation is more complicated [16]: in the $2+1$ dimensional setting where $h=h\left(x_{1}, x_{2}\right)$ the ADL mobility obtained by coarse-graining the step motion law is anisotropic, with eigenvalues $|\nabla h|^{-1}$ and 1 , and eigendirections normal and tangent to the steps. In the DL case, however, such complications are absent: the calculation in [16] supports the view that $M_{\mathrm{DL}}=1$.

The PDE (20) is somewhat formal, since we seem to be dividing by zero when $\nabla h=0$ - which is expected to occur, since (like the solution of the isoperimetric problem) the evolving height $h(x, t)$ is expected to have facets at its local minima and maxima. The PDE looks local, but actually it is nonlocal: the velocity of the facet edge depends on the size and shape of the facet (see [6] for a survey on singular diffusions like (20)).

When $M=1$ a fully rigorous understanding of this evolution is available, using the functional analysis of steepest-descent evolution, and the convexity of (21). Briefly: $h$ evolves by $H^{-1}$ steepestdescent for $E$, where $H^{-1}$ is dual to the space of mean-value-zero periodic functions endowed with the $H^{1}$ norm (see [8] for additional details and references to the literature). The evolution defined this way is the same as the one obtained by regularizing the singular term (e.g. replacing $|\nabla h|$ by $\left(|\nabla h|^{2}+\epsilon^{2}\right)^{1 / 2}$ then taking the limit $\left.\epsilon \rightarrow 0\right)$. It is also the same as the one obtained by implicit time-stepping in the limit $\Delta t \rightarrow 0$.

\subsection{Finite-time flattening when $M=1$ : heuristics}

Near the flattening time we expect $\nabla h$ to be small, so that $|\nabla h|^{3} \ll|\nabla h|$. Therefore we expect behavior similar to that obtained when $\beta_{3}=0$. Setting $\beta_{1}=1$ for simplicity, we are left to consider the $H^{-1}$ steepest descent for $\int|\nabla h| d x$, which formally solves

$$
h_{t}=-\Delta \operatorname{div}\left(\frac{\nabla h}{|\nabla h|}\right) .
$$


This evolution law has two scale invariances:

$$
t \rightarrow \lambda t, h \rightarrow \lambda h, x \rightarrow x \quad \text { and } \quad t \rightarrow \lambda^{4} t, h \rightarrow \lambda h, x \rightarrow \lambda x
$$

The first invariance shows that in its dependence on the initial data, the extinction time $T^{*}=T^{*}\left(h_{0}\right)$ is positively homogeneous of degree one, i.e. $T^{*}\left(\lambda h_{0}\right)=\lambda T^{*}\left(h_{0}\right)$ for $\lambda>0$. This suggests a flattening time estimate of the form

$$
T^{*} \leq C\left\|h_{0}\right\|_{X}
$$

for some function space $X$. The second invariance in (27) determines how the constant in (28) depends on the size of the period cell. In particular, it places restrictions on $X$ if we want the estimate to be scale-invariant (e.g. to be valid - with a fixed constant - when the period cell is a cube of any size).

Since any time can be viewed as the initial time, the heuristic estimate can be written

$$
\|h(t)\|_{X} \geq C^{-1}\left(T^{*}-t\right) \quad \text { for } t<T^{*} .
$$

Thus it captures (at the level of an inequality) the intuition that the solution should flatten linearly in time.

\subsection{Finite-time flattening: rigorous results}

The central accomplishment of [8] is a family of flattening time estimates for

$$
h_{t}=-\Delta \operatorname{div}\left(\frac{\nabla h}{|\nabla h|}+\beta_{3}|\nabla h| \nabla h\right)
$$

that are more or less of the form (28). Notice that (29) is our original equation (20) with $M=1$ and $\beta_{1}=1$.

The argument is relatively easy at the formal level. Multiplying (29) by $(-\Delta)^{-1} h$ and integrating by parts gives

$$
\begin{aligned}
\frac{1}{2} \frac{d}{d t}\|h\|_{H^{-1}}^{2}(t) & =\int h_{t}(-\Delta)^{-1} h d x \\
& =\int h \operatorname{div}\left(\frac{\nabla h}{|\nabla h|}+\beta_{3}|\nabla h| \nabla h\right) d x \\
& =-\int|\nabla h|+\beta_{3}|\nabla h|^{3} d x \\
& \leq-\int|\nabla h| d x
\end{aligned}
$$

For a non-scale-invariant estimate in dimension $n \leq 4$, we can finish by observing that

$$
\|h\|_{H^{-1}} \leq C \int|\nabla h| d x
$$


for periodic functions with mean value 0 , with a constant that depends on the period. Combining this with (30) gives

$$
\frac{d}{d t}\|h\|_{H^{-1}} \leq-C \text { for } t<T^{*}
$$

Since the right hand side is independent of $t$, we conclude that

$$
T^{*}\left(h_{0}\right) \leq C\left\|h_{0}\right\|_{H^{-1}}
$$

for any (periodic) initial condition $h_{0}$.

We are, however, especially interested in scale-invariant estimates, because the PDE is often used to simulate problems with complex or random initial data. In such settings, spatial periodicity is used numerically (with a sufficiently large period cell) as a scheme for minimizing "finite-size effects." The key, of course, is to replace (31) by a scale-invariant estimate. We showed in [8] that

$$
\|h\|_{H^{-1}} \leq C_{*}\left\|(-\Delta)^{-1} h\right\|_{W^{-1, p}}^{1-\theta}\left(\int|\nabla h| d x\right)^{\theta}
$$

for mean-value-zero periodic functions in $\mathbb{R}^{n}$, provided $1 \leq n \leq 4,1 \leq p \leq \infty$, and $1 / 2 \leq \theta \leq 1$ are related by

$$
1+\frac{n}{2}=\theta(n-1)+(1-\theta)\left(3+\frac{n}{p}\right) .
$$

Moreover, this estimate is scale-invariant, in the sense that while the constant $C_{*}$ depends on the shape of the period cell, it does not depend on its size.

To make use of (33) we must control the growth of $\left\|(-\Delta)^{-1} h\right\|_{W^{-1, p}}$. This is again easy at the formal level: since $\|\cdot\|_{W^{-1, p}}$ is a norm, we have

$$
\frac{d}{d t}\left\|(-\Delta)^{-1} h\right\|_{W^{-1, p}} \leq\left\|(-\Delta)^{-1} h_{t}\right\|_{W^{-1, p}}
$$

From the PDE (29) we have

$$
\left\|(-\Delta)^{-1} h_{t}\right\|_{W^{-1, p}}=\|z\|_{W^{-1, p}}
$$

with

$$
z=\operatorname{div}(\nabla h /|\nabla h|)+\operatorname{div}\left(\beta_{3}|\nabla h| \nabla h\right)=z_{1}+z_{2} .
$$

Since $\nabla h /|\nabla h|$ has magnitude less than or equal to 1 pointwise, we have

$$
\left\|z_{1}\right\|_{W^{-1, p}} \leq 1
$$

if the unit cell has volume 1 . As for $z_{2}$, we have

$$
\left\|z_{2}\right\|_{W^{-1, p}} \leq \beta_{3}\left\||\nabla h|^{2}\right\|_{L^{p}}
$$

which is controlled by the initial energy provided $p \leq 3 / 2$, using the fact that $E=\int|\nabla h|+\frac{1}{3} \beta_{3}|\nabla h|^{3}$ is decreasing in time. Our article [8] makes these arguments rigorous and uses them to show that

$$
T^{*} \leq \frac{1}{2-(1 / \theta)} C_{*}^{1 / \theta}\left\|(-\Delta)^{-1} h_{0}\right\|_{W^{-1, p}}^{(1 / \theta)-1}\left\|h_{0}\right\|_{H^{-1}}^{2-(1 / \theta)}
$$


for periodic solutions in $\mathbb{R}^{n}$ when $1 \leq n \leq 4,1 \leq p \leq 3 / 2$ and $1 / 2<\theta \leq 1$ are related by (34). The constant $C_{*}$ in (35) is the one from (33); in particular, it is scale-invariant. In the physical

case $n=2$, the relationship (34) reduces to $\left(2+\frac{2}{p}\right) \theta=1+\frac{2}{p}$. Any $p$ in the range $1 \leq p \leq 3 / 2$ is permitted, and $\theta(p)$ ranges between $\theta(1)=3 / 4$ and $\theta(3 / 2)=7 / 10$.

\subsection{Some open problems}

We have focused on the DL setting, where $M=1$ and (20) becomes (29). In this setting the evolution is $H^{-1}$ steepest descent for the surface energy (21). As we explained above, however, this is just a special case; physically, it is equally natural to consider $M(\nabla h)=(1+c|\nabla h|)^{-1}$ where $c$ is any constant. Alas, when $M$ is not constant even the well-posedness of the PDE seems unclear, since the evolution is no longer (to the best of our knowledge) steepest descent for (21) in any Hilbert space.

The ADL setting (when $M=|\nabla h|^{-1}$ ) has received substantial attention in the physics literature, both because some experiments are in this regime $[4,27]$ and because heuristic arguments like those of Section (3.2) are available in this case. Numerical simulation with sinusoidal initial data shows that $h_{\max } \sim \lambda^{-1}\left(T^{*}-t\right)^{1 / 2}$, where $\lambda$ is the spatial period [4, 27]. Interestingly, when the 1D, ADL evolution was simulated in [4] with more complex initial data modeling the decay of nanoripples on a $\mathrm{Cu}(001)$ surface, the result was different: the amplitude $\|h\|_{L^{2}}$ decayed with a linear rather than square-root law.

We expect solutions of (20) to flatten in finite time only when $\beta_{1}>0$. The behavior when $\beta_{1}=0$ has been considered heuristically and numerically. The expected behavior (see e.g. [4]) is that $h \rightarrow 0$ as $t \rightarrow \infty$, with $\|h\| \sim \tau / t$ in the DL setting and $\|h\| \sim e^{-t / \tau}$ in the ADL setting, where $\tau$ is a suitably defined characteristic time. Two-dimensional simulations with $\beta_{1}=0$ and the tensor mobility suggested by [16] reveal an interesting shape transition, in which biperiodic (but asymmetric) initial data become essentially one-dimensional [2].

Our discussion about the form of $M$ suggests that the PDE discussed in this section represents the continuum limit of the step model considered in Section 2. This conclusion is correct (at least formally) for a monotone step train separating two semi-infinite facets (see Appendix B of [1]). However it seems false in 1D for mean-value-zero initial data $[9,11]$, and it is definitely false in the radial context with an infinite cone as initial data [15]. The step and PDE models are consistent away from facets, but they treat the facets differently, providing (it seems) different evolution laws for the free boundary marking the edge of the facet. This does not mean that either model is wrong - but it raises the question, for a given physical system, which is more appropriate. Some insight is provided by [14] and [29], which derive equations like (20) by heuristically coarse-graining certain kinetic Monte Carlo models.

We have mentioned some numerical studies of (20). Those in $[4,26,27]$ use an interesting nonlinear Galerkin scheme whose numerical convergence has not yet been proved. However the convergence of a finite-element scheme for the DL equation (29) was recently examined in [13]. 


\section{References}

[1] H. Al Hajj Shehadeh, J. Weare, and R.V. Kohn, The evolution of a crystal surface: analysis of a $1 D$ step train connecting two facets in the ADL regime, submitted to Physica D

[2] A. Bonito, R. Nochetto, J. Quah, and D. Margetis, Self-organization of decaying surface corrugations: A numerical study, Phys. Rev. E 79, 2009, 050601.

[3] W.K. Burton, N. Cabrera, and F.C. Frank, The growth of crystals and the equilibrium structure of their surfaces, Philos. Trans. R. Soc. London Ser A 243, 1951, 299-358

[4] W.L. Chan, A. Ramasubramaniam, V.B. Shenoy, and E. Chason, Relaxation kinetics of nanoripples on $\mathrm{Cu}(001)$ surface, Phys. Rev. B 70, 2004, 245403

[5] P.W. Fok, R.R. Rosales, and D. Margetis, Facet evolution on supported nanostructures: the effect of finite height, Phys. Rev. B 78, 2008, 235401

[6] M.-H. Giga and Y. Giga, Very singular diffusion equations: second and fourth order problems, Japan J. Indust. Appl. Math. 27, 2010, 323-345

[7] J. Hager and H. Spohn, Self-similar morphology and dynamics of periodic surface profiles below the roughening transition, Surface Science 324, 1995, 365-372

[8] Y. Giga and R.V. Kohn, Scale-invariant extinction time estimates for some singular diffusion equations, to appear in Disc. Cont. Dyn. Sys. A

[9] N. Israeli, H.C. Jeong, D. Kandel, and J.D. Weeks, Dynamics and scaling of one-dimensional surface structures, Phys. Rev. B 61, 2000, 5698-5706

[10] N. Israeli and D. Kandel, Profile of a decaying crystalline cone, Phys. Rev. B 62, 1999, 59465962

[11] N. Israeli and D. Kandel, Decay of one-dimensional surface modulations, Phys. Rev. B 62, 2000, 12707-13717

[12] H.C. Jeong and E.D. Williams, Steps on surfaces: experiment and theory, Surface Science Reports 34, 1999, 171-294

[13] R.V. Kohn and H. Versieux, Numerical analysis of a steepest-descent model for surface relaxation below the roughening temperature, SIAM J. Numer. Anal., in press.

[14] J. Krug, H.T. Dobbs, and S. Majaniemi, Adatom mobility for the solid-on-solid model, Z. Phys. B 97, 1995, 281-291

[15] D. Margetis, P.W. Fok, M.J. Aziz, and H.A. Stone, Continuum theory of nanostructure decay via a microscale condition, Phys. Rev. Lett. 97, 2006, 096102 
[16] D. Margetis and R.V. Kohn, Continuum relaxation of interacting steps on crystal surfaces in $2+1$ dimensions, Multisc. Model. Simul. 5, 2006, 729-758

[17] D. Margetis and K. Nakamura, From crystal steps to continuum laws: behavior near large facets in one dimension, submitted to Physica D

[18] T. Michely and J. Krug, Islands, Mounds, and Atoms. Patterns and Processes in Crystal Growth Far from Equilibrium, Springer-Verlag, 2004

[19] M.V. Ramana Murty, Morphological stability of nanostructures, Phys. Rev. B 62, 2000, 1700417011

[20] W.W. Mullins, Theory of thermal grooving, J. Appl. Phys. 28, 1956, 333-339

[21] I.V. Odisharia, Simulation and analysis of the relaxation of a crystalline surface, $\mathrm{PhD}$ thesis, NYU, 2006

[22] M. Ozdemir and A. Zangwill, Morphological equilibration of a corrugated crystalline surface, Phys. Rev. B 42, 1990, 5013-5024

[23] A. Pimpinelli and J. Villain, Physics of Crystal Growth, Cambridge University Press, 1998

[24] A. Rettori and J. Villain, Flattening of grooves on a crystal surface: A method of investigation of surface roughness, J. Phys. I (France) 49, 1988, 257-267

[25] H. Spohn, Surface dynamics below the roughening transition, J. Phys. I France 3, 1993, 69-81

[26] V.B. Shenoy, A. Ramasubramaniam, and L.B. Freund, A variational approach to nonlinear dynamics of nanoscale surface modulations, Surface Science 529, 2003, 365-383

[27] V.B. Shenoy, A. Ramasubramaniam, H. Ramanarayan, D.T. Tambe, W-L. Chan, and E. Chason, Influence of step-edge barriers on the morphological relaxation of nanoscale ripples on crystal surfaces, Phys. Rev. Lett. 92, 2004, 256101

[28] L.-H. Tang, Flattening of grooves: from step dynamics to continuum theory, in Dynamics of Crystal Surfaces and Interfaces, P.M. Duxbury and T.J. Pence eds, Plenum Press, 1997, $169-184$

[29] D.D. Vvedensky, A. Zangwill, C.N. Luse, and M.R. Wilby, Stochastic equations of motion for epitaxial growth, Phys. Rev. E 48, 1993, 852-862 